\title{
Orion Handling Qualities During ISS Proximity Operations and Docking
}

\author{
John-Paul Stephens ${ }^{1}$ \\ Lockheed Martin Exploration \& Science Solutions, Houston, TX, 77058 \\ Gordon A. Vos ${ }^{2}$ \\ Wyle IS\&E, Houston, TX, 77058 \\ Karl D. Bilimoria ${ }^{3}$, Eric R. Mueller ${ }^{4}$ \\ NASA Ames Research Center, Moffett Field, CA, 94035 \\ Jack Brazzel $^{5}$, Pete Spehar ${ }^{6}$ \\ NASA Johnson Space Center, Houston, TX, 77058
}

\begin{abstract}
NASA's Orion spacecraft is designed to autonomously rendezvous and dock with many vehicles including the International Space Station. However, the crew is able to assume manual control of the vehicle's attitude and flight path. In these instances, Orion must meet handling qualities requirements established by NASA. Two handling qualities assessments were conducted at the Johnson Space Center to evaluate preliminary designs of the vehicle using a six degree of freedom, high-fidelity guidance, navigation, and control simulation. The first assessed Orion's handling qualities during the last $20 \mathrm{ft}$ before docking, and included both steady and oscillatory motions of the docking target. The second focused on manual acquisition of the docking axis during the proximity operations phase and subsequent station-keeping. Cooper-Harper handling qualities ratings, workload ratings and comments were provided by 10 evaluation pilots for the docking study and 5 evaluation pilots for the proximity operations study. For the docking task, both cases received $90 \%$ Level 1 (satisfactory) handling qualities ratings, exceeding NASA's requirement. All ratings for the ProxOps task were Level 1. These evaluations indicate that Orion is on course to meet NASA's handling quality requirements for ProxOps and docking.
\end{abstract}

\section{Introduction}

NASA's Constellation Program had a goal to provide sufficient and consistent handling qualities for manual controllable operations across all new Constellation vehicles, which included the Orion spacecraft. The Orion spacecraft has been designed to rendezvous and dock with other Constellation vehicles in low earth orbit and beyond, including the International Space Station (ISS). Although Orion has autonomous docking capabilities, it is expected that final approach and docking operations with the ISS will initially be performed manually. Furthermore, NASA's Orion Program includes a specific system requirement ${ }^{1,2}$ that Orion must provide manual control for proximity operations and docking, while other human rating and integration requirements mandated that

\footnotetext{
${ }^{1}$ Human Factors Design Engineer, P.O. Box 58487 LM-2, Mail Stop B2A; John-Paul.Stephens@lmco.com.

${ }^{2}$ Senior Human Factors Engineer, 1290 Hercules Drive; gordon.vos@wyle.com. Senior Member AIAA

${ }^{3}$ Aerospace Engineer, Flight Trajectory Dynamics and Controls Branch, Mail Stop 210-10; Karl.Bilimoria@nasa.gov. Associate Fellow AIAA

${ }^{4}$ Aerospace Engineer, Flight Trajectory Dynamics and Controls Branch, Mail Stop 210-10; Eric.Mueller@nasa.gov. Senior Member AIAA

${ }^{5}$ Aerospace Engineer, GN\&C Autonomous Flight Systems Branch, Mail Code EG6; Jack.Brazzel@nasa.gov. Senior Member AIAA

${ }^{6}$ Aerospace Engineer, GN\&C Autonomous Flight Systems Branch, Mail Code EG6; Peter.T.Spehar@nasa.gov.
} 
handling qualities ratings required during manual control must be Level 1 during nominal operations (such as docking).

As published in their seminal 1969 paper "The Use of Pilot Rating in the Evaluation of Aircraft Handling Qualities," authors Cooper and Harper established the definition of handling qualities as "those qualities or characteristics of an aircraft that govern the ease and precision with which a pilot is able to perform the tasks required in support of an aircraft role." ${ }^{3}$ What the pilot feels (vehicle response), what the pilot sees (out-the-window \& displays), and what the pilot touches (input devices) are handling qualities factors.

Traditionally assessed using military-trained test pilots, handling qualities have been an important factor in spacecraft design and development from the earliest days of the space program. This legacy is demonstrated by NASA's use of test pilots as its piloting astronauts starting with Mercury and continuing with Gemini, Apollo, and Space Shuttle. Some of the initial work to support manual piloting of spacecraft addressed rendezvous and docking operations, ${ }^{4-8}$ manual control modes, ${ }^{9-14}$ use of video for docking operations when direct view is not possible, ${ }^{14,15}$ visual aids to enable direct view, failed sensors, ${ }^{9,11-13,15}$ flight control parameters, ${ }^{16}$ bounding of attitude oscillation, ${ }^{10}$ failed propulsion components such as jets or thrusters, ${ }^{9}$ and the overall assessment of handling qualities for Gemini and Apollo. ${ }^{9,12,14}$ Much of what was learned in these early programs pertaining to manual piloting was applied to the Space Shuttle Program. ${ }^{7}$ Subsequently, the design of the Orion systems enabling manual piloting during proximity operations and docking was largely influenced by Shuttle-to-Mir and Shuttle-to-ISS development and operational experience. ${ }^{17-18}$

A series of evaluations were conducted at the Johnson Space Center to determine the handling qualities (HQ) of the Orion spacecraft during different docking and proximity operations conditions using the Cooper-Harper rating scale. This paper addresses the specifics of the handling qualities methodology, simulation environment, scenarios flown, data collection tools, and subject ratings and comments from these evaluations

\section{Methodology}

This evaluation was part of the Orion Spacecraft Handling Qualities Assessment. The objective of the assessment was to evaluate the handling qualities of the Orion spacecraft iteratively throughout the Orion design phase. This iterative assessment process (see Figure 1) is intended to be conducted early in the design process and repeated as necessary in order to identify and mitigate potential handling qualities problems so that engineering modifications can be made to assure requirement compliance during the verification phase of the Orion project. This paper discusses the current handling qualities results of the Rendezvous, Proximity Operations, and Docking (RPOD) portion of this assessment. The goal of the evaluation was to determine if the Guidance, Navigation, and Control (GN\&C) design and vehicle configuration can meet spacecraft handling performance requirements (i.e. thruster configuration, sufficient control authority, etc.) set forth by NASA.

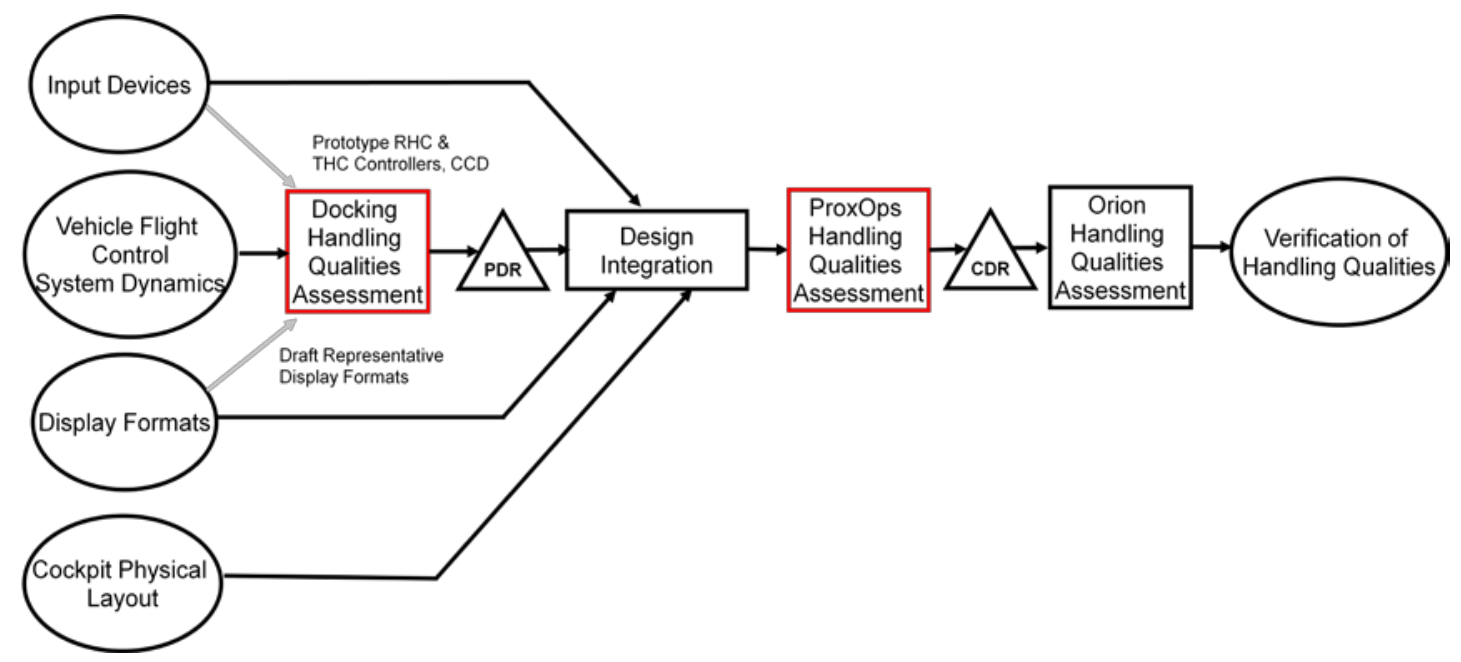

Figure 1. Orion handling qualities evaluations testing program 
The RPOD portion of the handling qualities assessment consists of two separate evaluations: one focusing on final approach for docking Orion to ISS (Docking evaluation) and the second focusing on acquisition of the docking axis (ProxOps evaluation). The Docking and ProxOps evaluations were performed during the summers of 2008 and 2010 respectively. The planning and execution of these two evaluations were a collaborative NASA and Lockheed Martin (LM) endeavor which included multiple engineering disciplines from each. Key roles in the evaluation were performed by the Astronaut Office, Guidance, Navigation and Control (GN\&C) Engineers, Human Factors Engineers, Simulation Engineers with consulting from Ames Research Center (ARC) and Langley Research Center (LaRC) Spacecraft Handling Qualities (SHaQ) group.

Astronauts served in three different roles during the development and execution of these evaluations. Some astronauts were involved in the planning and development, some participated in the dry run activities and others were involved as evaluation pilots in the evaluation. Only astronauts who had not participated in the development or dry run activities served as evaluation pilot. For each of the two evaluations, two shuttle commanders with test piloting experience served as dry run evaluators. Ten astronauts evaluated the Docking scenario, while five assessed the ProxOps scenario. All evaluation pilots were experienced astronauts and the majority of them (11 of 15) were experienced military test pilots. All evaluation pilots were briefed on the evaluation, informed of their rights as subjects in the evaluation, and asked to sign informed consent forms. The protocol for these assessments was approved by NASA's Committee for the Protection of Human Subjects (NASA's internal Institutional Review Board (IRB) for research involving human subjects).

Preceding the evaluations was a period of requirements review in which the mandate for manual control was assessed, as well as a period of task review in which we looked at roughly 100 potential manual control tasks during a mission of the Orion capsule to ISS and back. These 100 manual control tasks were then reduced to about 30 based on the likelihood of them being needed or allowing for manual control within vehicle margins, and finally down to 11 key tasks that the team felt were the core manual control tasks that the program needed to evaluate and verify. The evaluation flow can be seen in Figure 2.

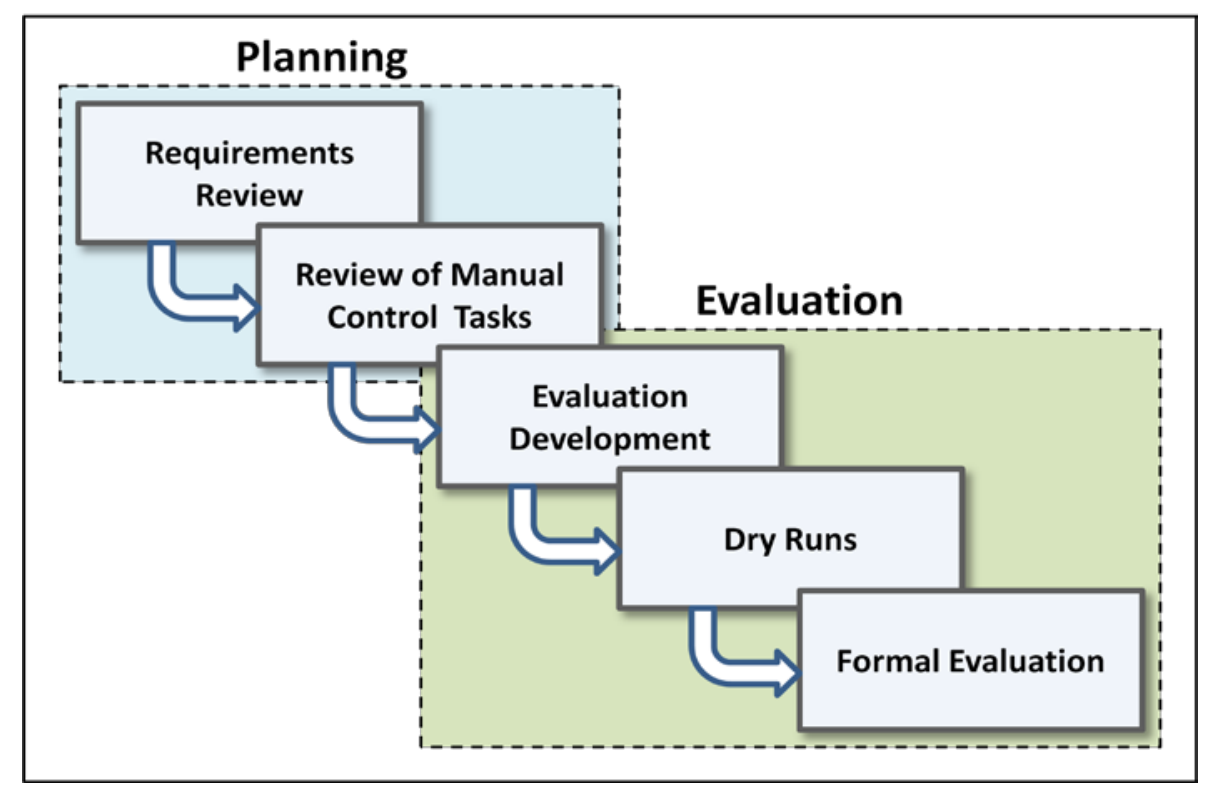

Figure 2. Evaluation flow

Evaluation planning then began, with each evaluation broken into three distinct phases: evaluation development, dry runs, and formal evaluation. The evaluation development phase included discussion and assessment of what types of manual control scenarios to investigate, identification of appropriate performance metrics for each scenario, and design/development of simulations for candidate scenarios. Once the scenarios were simulated and the metrics derived, dry run assessments were used to evaluate the scenario simulations and evaluation procedures. Feedback from the dry run evaluators was used to improve subject briefing packages, scenario-specific piloting metrics, and evaluation time estimates. At the start of each formal evaluation session, the subject received an Orion Spacecraft Handling Qualities Assessment overview, a briefing on the 1969 Cooper- 
Harper $(\mathrm{CH})$ rating scale, and a scenario-specific briefing. Following the briefings, the subject was given unlimited familiarization time in the simulator to become comfortable with the controls, displays, flight tasks being evaluated, and to be exposed to the different cases within the scenario.

Ratings were not collected during the familiarization runs and the subject was allowed to experiment with different flight techniques during each run. Once the subject indicated comfort with the scenario, the subject would perform a case-specific practice run for the particular case under evaluation. After completing the practice run, the subject would perform two data runs with an option for a third data run. If the subject was satisfied with his practice run performance, the subject could elect to use the practice run as the first data run. If the subject was dissatisfied with the practice run, an additional practice run was offered. CH ratings were collected after the completion of all data runs for each case. Once the subject's $\mathrm{CH}$ ratings and comments were collected, the subject would start practice runs on the next case. Objective data (described later) were collected on every familiarization, practice, and data run, but only subjective data collected during the data runs will be discussed in this report. Subject comments were collected throughout the entire evaluation, including during familiarization runs. Cases and initial conditions were randomly assigned to each subject and every subject evaluated every case (but not every subject saw every initial condition). After the completion of all cases, the subject was debriefed on the results of the session. Before any scripted questions were asked, the subject was asked for observations and comments on the scenario. The pilot was then asked scripted questions from a questionnaire as well as unscripted follow-up questions from the test team.

\section{Simulation and Facilities}

The simulation used the Advanced NASA Technology Architecture for Exploration Studies (ANTARES), ${ }^{19-20}$ which is the NASA high-fidelity, six degrees-of-freedom simulation used for Orion GNC algorithm development and analysis. It has been designed to support a wide variety of requirements assessments, design trades, and operator-in-the-loop evaluations for the Orion Crew Module (CM). ANTARES is based on a Common Model Library architecture and the Trick simulation tool kit which leverages off the significant simulation development conducted by several organizations across JSC, including the Aeroscience and Flight Mechanics Division, the Automation, Robotics and Simulation Division and the Mission Operations Directorate. In 2008, it won the JSC Exceptional Software Award, the NASA Exceptional Technology Achievement Medal, and the NASA Agency Software of the Year. It is used for Orion GN\&C algorithm development and analysis. Although primarily a batch simulation, ANTARES can be used for real-time pilot-in-the-loop evaluation with a Translational Hand Controller (THC), Rotational Hand Controller (RHC), notional displays for piloting cues, flight control system configuration (pilot selected translational authority: coarse/fine), and real-time graphics for simulated out-the-window and docking camera views. ANTARES was used for development of the manual control algorithm prototypes used in the selected scenarios, determining the Cooper-Harper criteria, and specific cases to be used for the evaluations. Current sets of mass properties, initial vehicle state, aerodynamics, atmospheric, and navigation sensor models were included into the ANTARES simulation. Nominal and dispersion sets for all important parameters were implemented.

NASA's Reconfigurable Operational Cockpit (ROC) facility was used for both assessments to provide the Orion cockpit environment. The three Orion display units in the ROC are capable of showing Orion displays, overlays, and docking camera views. The ROC (see Figure 3) also provides out-the-window views for situational awareness using image projection. 


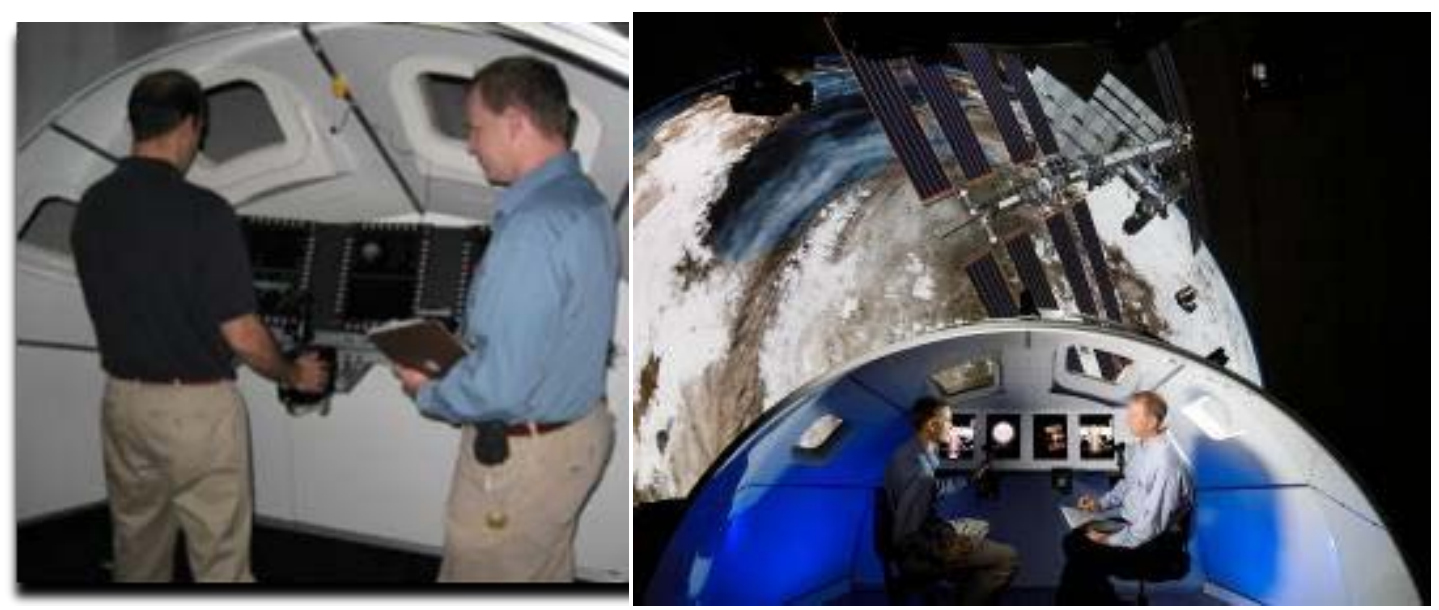

Figure 3. The ROC facility. The left shows the actual setup (illuminated by the camera flash) and the right shows the type of out-the-window scene generation capability that was used.

\section{Cooper-Harper Rating Scale}

The assessment of handling qualities was performed using the 1969 Cooper-Harper rating scale. ${ }^{3}$ The CooperHarper rating scale is the most commonly used handling qualities assessment tool and is required by NASA for spacecraft human rating. ${ }^{16}$ The Cooper-Harper decision tree (Figure 4) begins with an assessment of the vehicle's "Adequacy for Selected Tasks or Required Operation" in which the evaluation pilot decides if the performance achieved in a piloting run was desired, adequate or uncontrollable (these are adjectives associated with performance and used as anchors at various locations within the scale). Performance is defined as the precision of control with respect to flight vehicle movement that a pilot is able to achieve in performing a task. Adequate performance means that the end result obtained was sufficient for successful completion of the required operation or task. Desired performance means that the end result obtained exceeded the level of performance needed for successful completion of the required operation or task. Since these terms (desired/adequate performance) are involved in the core decision logic of the scale, the $\mathrm{CH}$ performance metrics associated with their definition are key drivers of the rating process. Following the initial determination of operational adequacy, the evaluation pilot subject then proceeded to the right of the scale into the defined categories of Level 1, 2 or 3. Next the evaluation pilot reviewed the flight vehicle characteristics, the demands on the pilot, and determined the final rating. The Cooper-Harper protocol allows for half-ratings within a level (1.5, 2.5, 4.5, 5.5, 7.5, 8.5), but does not allow half-ratings across levels (3.5, $6.5,9.5)$.

In handling qualities experiments, evaluation pilots are typically asked to make a composite assessment of the overall performance across all formal evaluation runs for a test configuration. This assessment takes into account not just the quantitative evaluation of the performance at the end point (e.g., hard dock) but also a qualitative evaluation of the manner in which the vehicle gets to the end point. This overall assessment of desired, adequate, or inadequate performance is utilized for traversing the decision tree in the Cooper-Harper chart. ${ }^{3}$ Pilots use the Cooper-Harper scale to assign handling qualities ratings from 1 (best) to 10 (worst) based on their assessment of task performance and required compensation. It is an ordinal, non-linear scale, such that the difference between ratings 1 and 2 is not the same as the difference between ratings 3 and 4, thus the data collected using the scale must be assessed using non-parametric methods which lack distributional assumptions of normality or linearity. Additionally, the ratings are categorized into levels, such that ratings of 1, 2, and 3 on the Cooper-Harper scale correspond to Level 1 handling qualities, which are a general requirement for normal operations of flight vehicles. Desired performance is necessary, but not sufficient, for Level 1 ratings. Ratings of 4, 5, and 6 correspond to Level 2 which may be acceptable for some off-nominal conditions, while ratings of 7, 8, and 9 correspond to Level 3 which is acceptable only for transition to a safe mode after a major failure or disturbance. 


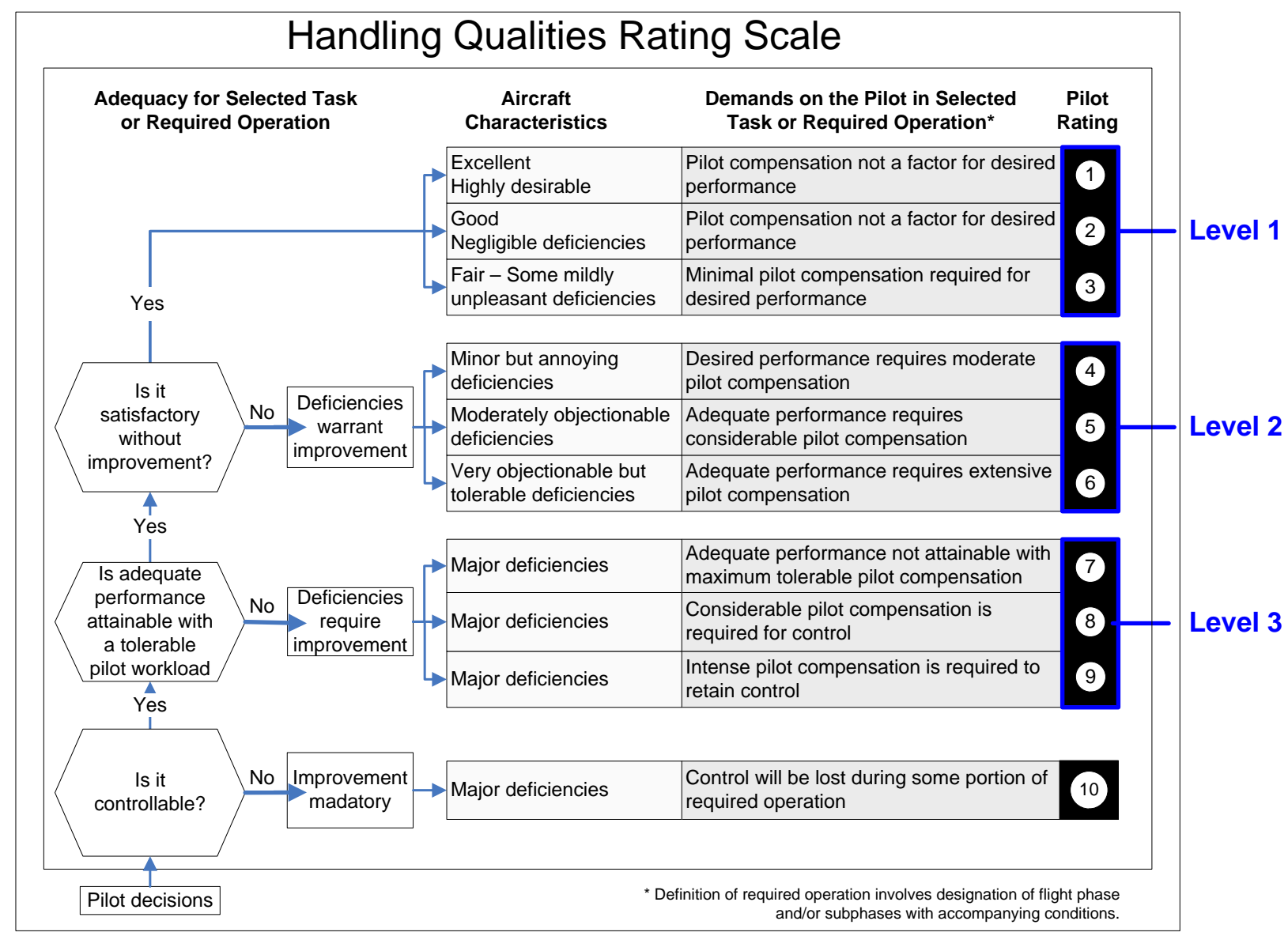

Figure 4. Cooper-Harper Rating Scale reproduced from NASA TN D-5153 ${ }^{3}$

\section{Workload}

The NASA Task Load Index (TLX) ${ }^{21}$ and Bedford Pilot Workload Rating Scales ${ }^{22-23}$ were also used for the Docking and ProxOps scenarios respectively. The reason the workload measures were not consistent between the evaluations is because the NASA requirements governing workload changed between the two evaluations. During the earlier Docking Evaluation, NASA required TLX, but later, prior to the ProxOps evaluation, the NASA requirements for workload changed to the Bedford Workload Scale. Workload was not of the main objective of these evaluations, but is an important measure that can contribute to the handling qualities ratings and thus is useful to understand.

The NASA Task Load Index is a weighted average of six workload components: physical demand, mental demand, temporal demand, performance, effort, and frustration. ${ }^{21}$ The relative weighting of these six components for the flying task was determined by a pilot questionnaire at the end of the experiment. Each component is assigned a rating between 0 and 100. Unlike the Cooper-Harper or Bedford scales, there are no descriptors associated with TLX component ratings; there is only a low/high anchoring on the ends of the scale. A rating of zero corresponds to low, and a rating of 100 corresponds to high. The rating for performance is subtracted from 100 prior to computing the weighted average.

The Bedford Pilot Workload Rating Scale (Figure 5) was utilized to assess the workload associated with the ProxOps manual control task. Workload is an important measure that can contribute to the handling qualities ratings and thus is useful to understand. The Bedford Rating Scale was administered exactly like the Cooper-Harper Scale. Evaluation pilots were asked to start at the bottom and walk through each logic gate. Unlike $\mathrm{CH}$, Bedford is not broken up into levels and half ratings between decision gates are acceptable (e.g. ratings of 3.5 or 6.5 ). ${ }^{22-23}$ 


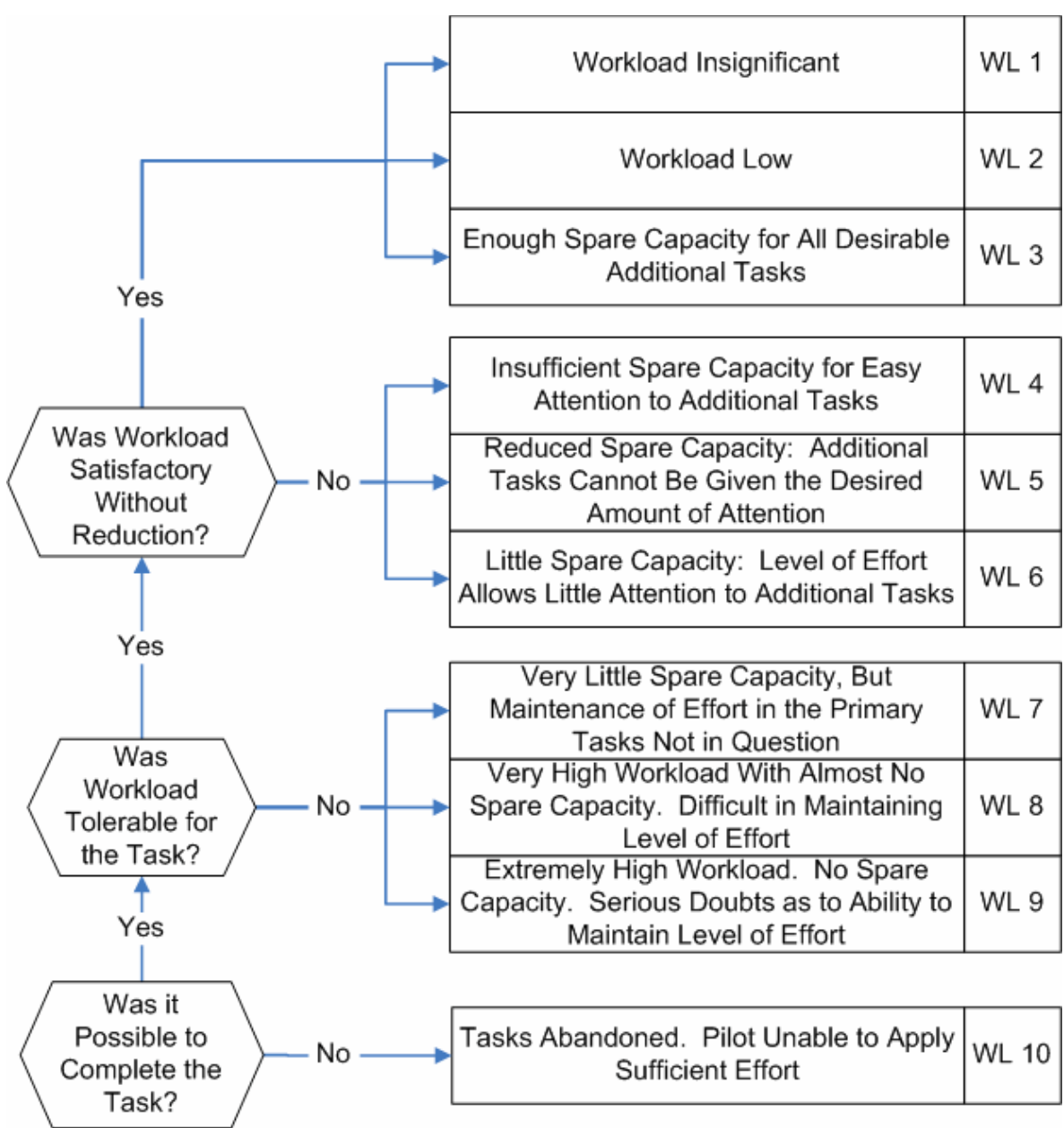

Figure 5. Bedford Pilot Workload Rating Scale ${ }^{23}$

\section{Additional Data}

Several objective simulation variables were monitored and collected to facilitate interpretation of subjective handling qualities ratings and comments. The ANTARES data relevant to the simulation itself (e.g. vehicle state parameters, flight path information, etc.) were electronically recorded. The collection of these variables was programmed into the simulation by ANTARES programmers and GN\&C specialists. The data collected included objective performance criteria used in evaluation for determining the adequacy of performance for Cooper-Harper rating purposes. Relevant data collected includes:

- $\quad$ Timing information (duration of runs, time stamps for hand controller inputs)

- $\quad$ Hand controller inputs (type, direction, magnitude, frequency)

- $\quad$ Control system mode (pilot selected translational authority: coarse/fine)

- $\quad$ Trajectory and performance

o Vehicle relative trajectory (position and velocity)

o Vehicle orientation (attitude and rotational rates)

o Vehicle control errors

o Propellant used/remaining

Additional subjective data was collected by the test conductor. Evaluation pilots' verbal comments were digitally recorded throughout the evaluation and were also collected by test team note takers. The pre-brief was the 
only portion of the evaluation where evaluation pilots' comments were not recorded. While video of the evaluation was transmitted to test team members monitoring the evaluation from outside of the ROC, the video was not recorded.

\section{Assumptions and Constraints}

The primary outcome of this evaluation was an assessment of Cooper-Harper ratings associated with Orion's handling qualities, given the evaluation's constraints and assumptions. The computer simulation models, derived from ANTARES version 9.2.0, were high fidelity and accurate with regards to the physics of Orion under various flight conditions and use of actual Orion GN\&C flight control algorithms. For these manually piloted scenarios, relative-navigation's main purpose was to drive trajectory data on the crew displays. However, due to navigation model certification schedules, a decision was made to use a perfect relative navigation model instead of the Orion baseline relative navigation flight software. The perfect relative navigation model simply replaces internal relative navigation state estimates (position and velocity) with the true state generated from the simulation equations of motion, thus producing a perfect navigation output (no noise or bias errors). To be certain that this decision would not impact the study results, a visual comparison of the display output of both of these navigation models (perfect and Orion baseline) was conducted. The comparison showed no noticeable difference to engineer observers so the fidelity of the model was not expected to influence the evaluation results. Also, the GN\&C team's analysis of propellant slosh effects on flight control performance concluded that propellant slosh had an insignificant affect on flight control; therefore, a propellant slosh model was not included for this study.

Space suits, gloves, and helmets were not employed in these evaluations. Evaluation pilots wore typical office clothing. Nominally, Orion flight crew would be fully suited for all scenarios evaluated, but suits were not incorporated into the evaluation primarily due to suit availability and resources. Future HQ evaluations will consider using suited evaluation pilots as it is anticipated this will be required for final vehicle verification.

\section{$\underline{\text { Scenarios }}$}

The initial Orion HQ evaluation (Docking Scenario) examined docking Orion to the ISS. This scenario demonstrated the translational motion handling qualities of Orion. During the Docking scenario, two different cases were evaluated. The first case was a docking approach to a fixed-attitude ISS (Stable ISS), with Orion initializing with relative position dispersions (a 4 degree offset, which is not nominal, but added some stress to the piloting task to expose any underlying handling qualities issues) and a closing rate of approximately $0.1 \mathrm{ft} / \mathrm{sec}$. Previous shuttle docking operations have shown the ISS motion to be imperceptible by the shuttle astronauts. For this reason, the ISS was considered a stable platform during the first docking scenario. The second docking case was identical to the first, except that the attitude motion of the ISS was modeled to simulate a highly perturbed case $( \pm 1$ degree deadband per axis and $\pm 0.01 \mathrm{deg} / \mathrm{sec}$ rate deadband per axis) with the ISS attitude-hold control function engaged (Perturbed ISS). For both cases, evaluation pilots started each run on final approach at a docking port-to-port range of $20 \mathrm{ft}$. Evaluation pilots used the THC in pulse mode with cues from the docking camera image, window views, and range and range rate data presented on the Orion display units. As in the actual Orion design, the attitude of the vehicle was held by the automated flight control system at \pm 0.5 degree deadband per axis. Several error sources were modeled including Reaction Control System (RCS) jet angular and position misalignment, RCS thrust magnitude uncertainty, RCS jet force direction uncertainty due to self plume impingement, and Orion center-ofmass uncertainty. Both Docking cases used the same objective performance criteria, listed in Table 1. Table 2 provides the translational pulse size settings for this scenario. Evaluation pilots were allowed to switch between these settings to achieve their desired pulse size. The Docking cases and initial conditions were presented to evaluation pilots in a random order.

Evaluation pilots used two displays (Figure 6 and 7) to perform the docking procedure: a view from the docking camera with cross-hairs reticle overlay pointing outward along the centerline of the Orion docking port (Orb Cam) and a Primary Flight Display (Orb PFD). The centerline camera gave the evaluation pilots a visual perspective of the docking port, providing lateral translational cues, while the PFD provided the evaluation pilots with a digital indication of its current relative position and velocity. 
Table 1. Cooper-Harper performance criteria at docking port contact for Docking scenario

\begin{tabular}{|c|c|c|}
\hline Criteria & Desired & Adequate \\
\hline Lateral Offset & 1 inch from center & 2 inches from center \\
\hline Axial Closure Rate & $0.1 \mathrm{ft} / \mathrm{s} \pm 0.01 \mathrm{ft} / \mathrm{s}$ & $0.1 \mathrm{ft} / \mathrm{s} \pm 0.02 \mathrm{ft} / \mathrm{s}$ \\
\hline
\end{tabular}

Table 2. Translational pulse size settings for the Docking scenario

\begin{tabular}{|c|c|c|}
\hline Translational Pulse Sizes & Stable ISS & Perturbed ISS \\
\hline Coarse & $0.01 \mathrm{ft} / \mathrm{s}$ & $0.03 \mathrm{ft} / \mathrm{s}$ \\
\hline Fine & $0.005 \mathrm{ft} / \mathrm{s}$ & $0.01 \mathrm{ft} / \mathrm{s}$ \\
\hline
\end{tabular}

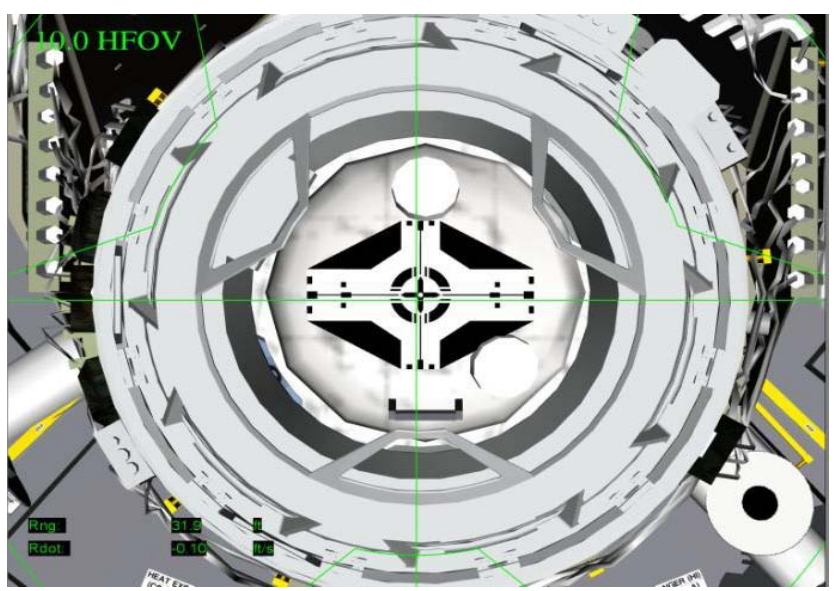

Figure 6. Engineering display of the docking camera view used for docking

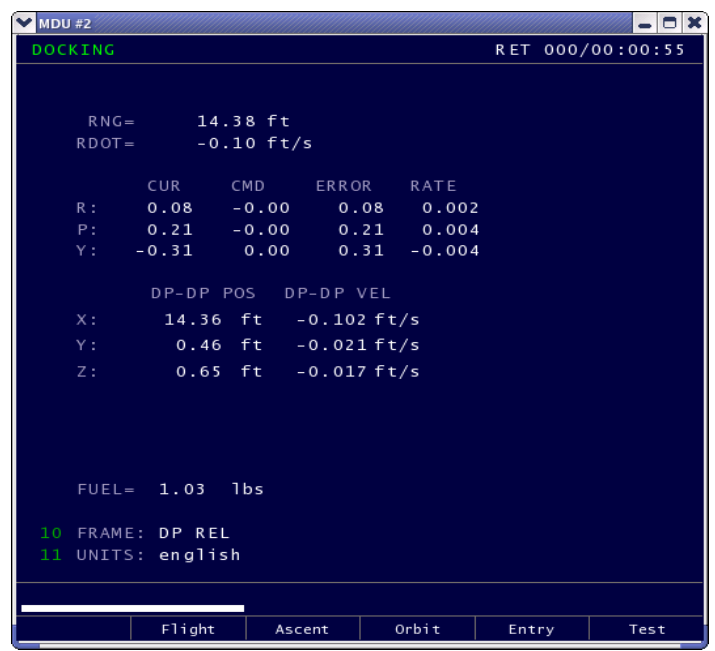

Figure 7. Engineering PFD showing docking rates and offsets

The second evaluation (ProxOps scenario) evaluated the handling qualities of Orion while acquiring the docking axis. This scenario demonstrates the coarse translational control authority by arresting the lateral motion during the Acquisition of Docking Axis (ADA) maneuver on the docking axis (see Figure 8). Evaluation pilots used the THC in a pulse mode to acquire the docking axis, and then arrest closure rate and perform station-keeping. As with both the Docking scenario, the Orion's attitude was automatically controlled by the flight control system. The target (ISS) was in a Torque Equilibrium Attitude of zero degrees of yaw and roll and negative seven degrees of pitch with respect to the Local Vertical Local Horizontal frame. The ProxOps scenario modeled several error sources including RCS jet angular misalignment, RCS jet position, RCS thrust uncertainty, RCS jet force direction uncertainty due to self plume impingement, Orion center of mass uncertainty, initial relative state dispersions (Orion with respect to ISS position, velocity, and attitude), and ISS attitude control deadbanding (within the range used for the Perturbed ISS case from the Docking Scenario). 


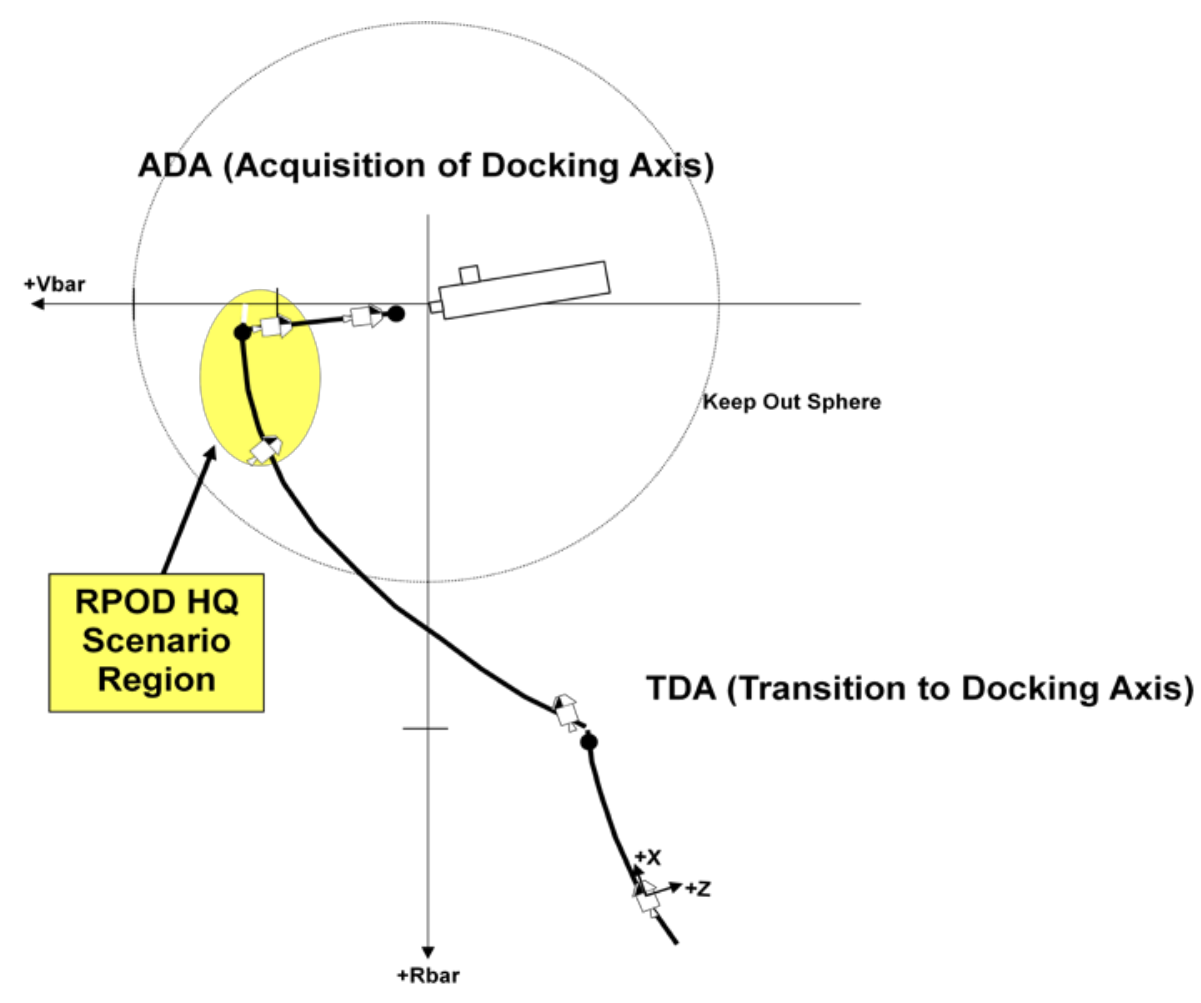

Figure 8. ProxOps scenario flight profile

Evaluation pilots were provided with three displays to perform the ProxOps task; a view from the docking camera pointing outward along the centerline of the Orion docking port (Orb Cam) similar to Figure 3, an orbit relative trajectory (Orb Traj) display, and an updated orbit Primary Flight Display (Orb PFD). The centerline camera gave the evaluation pilots a visual perspective of the docking axis acquisition maneuver, while the Orb Traj provided the evaluation pilots with a graphical representation of Orion's past, current, and future trajectory relative to the ISS, as well as a digital indication of its current relative position and velocity. The Orb PFD provided the subject with a countdown clock that indicated when Orion would be in docking attitude (In Att, MM:SS), but many of the other features present on the display were not operational.

The ProxOps scenario only contained one case with several different initial conditions (ICs). The scenario would initialize about two minutes prior to Orion arrival on the docking axis at a range of $300 \mathrm{ft}$ with a closing rate of approximately 0.50 feet per second. During the acquisition of the docking axis, the Orion flight control system would automatically maneuver Orion's initial attitude to the required docking axis attitude with a pitch rate of 0.13 degrees per second. Depending on the initial Orion attitude, Orion would arrive, positionally, on the docking axis before achieving the desired docking axis attitude (early case), or arrive at the docking axis after completing the attitude maneuver (late case). Several of the initial conditions included out-of-plane dispersions. In all, the ProxOps scenario contained five different ICs that were randomly presented to the evaluation pilots. The available ICs were:

- $\quad$ Arrive on time without an out of plane offset (nominal)

- $\quad$ Arrive Early with +y out-of-plane

- $\quad$ Arrive Early with -y out-of-plane

- $\quad$ Arrive late with +y out-of-plane

- $\quad$ Arrive late with -y out-of-plane 
Table 3 provides the translational pulse size settings for the ProxOps scenario. Evaluation pilots were allowed to switch between these settings to achieve their desired pulse size. Piloting techniques were not provided to the evaluation pilots for the ProxOps scenario. Evaluation pilots developed their own piloting techniques during their familiarization runs. Evaluation pilots were asked to acquire the docking axis and then perform station keeping (arrest closure rate). The Cooper-Harper performance criteria for this scenario are listed in Table 4. The desired and adequate criteria for position were developed by using a 2 degree and 3 degree corridor at a range of 250 feet respectively. It should be noted that these corridors are not requirements based or operationally based, but were chosen for this evaluation based on engineering judgment from NASA GN\&C specialists. The criteria for relative velocity were selected to provide a reasonably small value that effectively results in station-keeping. Evaluation pilots could use the digital display of their position and velocities on the Orb Traj display to determine if they were meeting the performance criteria.

Table 3. Translational pulse size settings for the ProxOps scenario

\begin{tabular}{|c|c|}
\hline Translational Pulse Sizes & ProxOps \\
\hline Coarse & $0.05 \mathrm{ft} / \mathrm{s}$ \\
\hline Fine & $0.01 \mathrm{ft} / \mathrm{s}$ \\
\hline
\end{tabular}

Table 4. ProxOps Cooper-Harper performance criteria

\begin{tabular}{|c|c|c|c|c|}
\hline \multirow{5}{*}{ Desired } & \multicolumn{4}{|c|}{ Docking Port to Docking Port } \\
\hline & \multicolumn{2}{|c|}{ Position (ft) } & \multicolumn{2}{|c|}{ Velocity (ft/s) } \\
\hline & $\mathrm{X}$ & -- & $\mathrm{Xd}$ & $<0.03$ \\
\hline & $\mathrm{Y}$ & $<9$ & $\mathrm{Yd}$ & $<0.03$ \\
\hline & $\mathrm{Z}$ & $<9$ & $\mathrm{Zd}$ & $<0.03$ \\
\hline \multirow{3}{*}{ Adequate } & $\mathrm{X}$ & -- & $\mathrm{Xd}$ & $<0.05$ \\
\hline & $\mathrm{Y}$ & $<13$ & Yd & $<0.05$ \\
\hline & $\mathrm{Z}$ & $<13$ & $\mathrm{Zd}$ & $<0.05$ \\
\hline
\end{tabular}

\section{Results}

Appropriate statistical methods for analyzing and displaying Cooper-Harper ratings are constrained by the ordinal nature of the rating scales. As there is not a linear or other parametric relationship implied across the rating spectrum, only nonparametric ordinal methodologies are acceptable for analysis of Cooper-Harper ratings data. For this reason the data analyses have focused upon fundamental graphical and numerical assessment techniques such as frequency scatter plots. Likewise, due to the possibly non-linear of latent traits assessed via use of the TLX and Bedford scales, workload data was examined using graphical techniques such as multi-axis plots.

Handling qualities ratings for the Docking and ProxOps scenarios are presented in Figure 9 where the size of the bubble for a given rating value indicates the number of evaluation pilots who assigned that rating, while the star symbol indicates the median rating for that category. For the two Docking cases, nine of ten evaluation pilots gave each case a Level 1 rating (rating of 1, 2, or 3) and one evaluation pilot gave each case a Level 2 rating. 


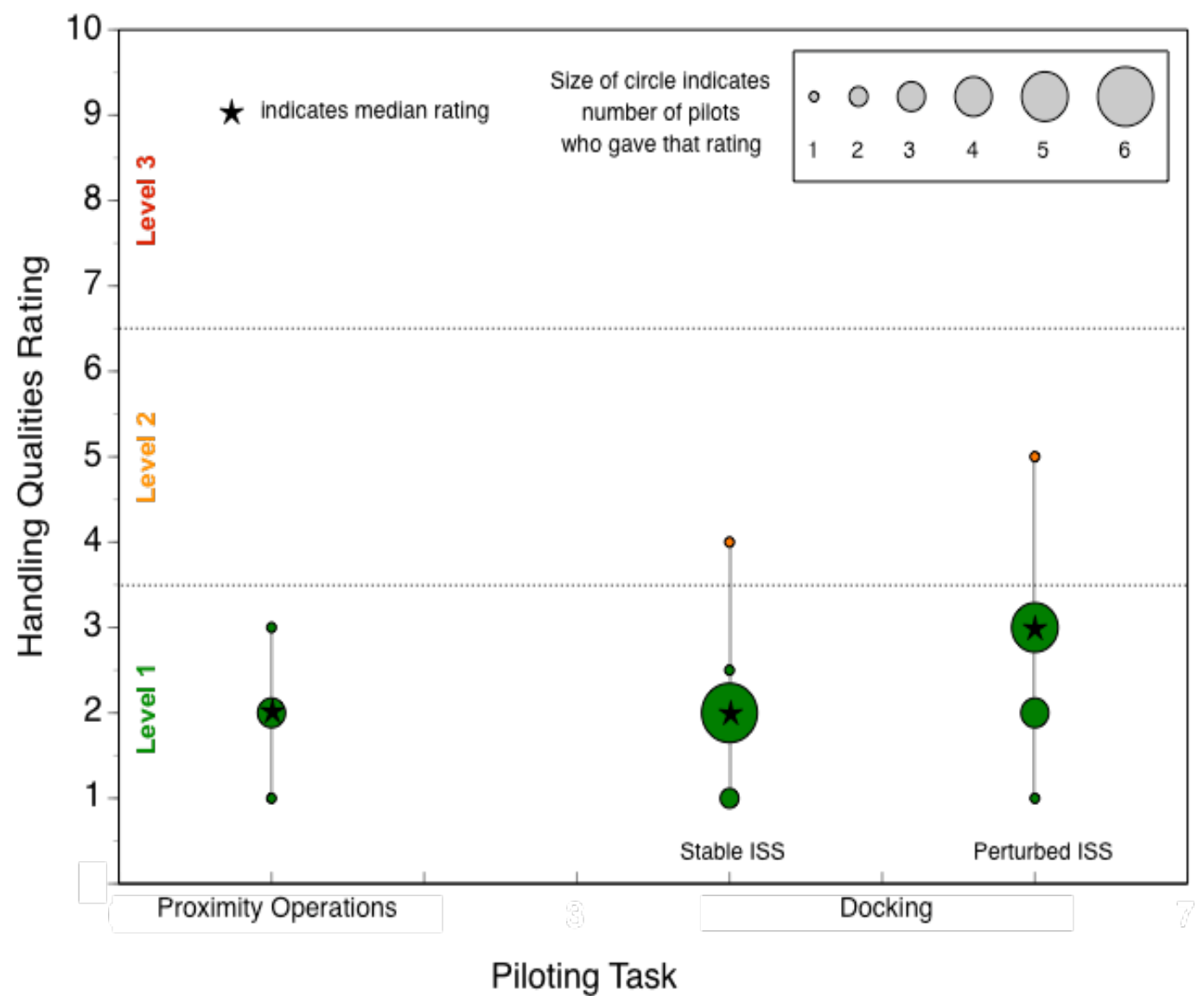

Figure 9. Handling qualities ratings for ProxOps and Docking tasks

Every run for the docking scenario had 'desired' performance for the $\mathrm{CH}$ metrics listed in Table 1 . The evaluation pilots used their individual performance metric results to help them determine their Cooper-Harper Ratings. Subject comments for the stable ISS docking scenario were fairly consistent, with evaluation pilots noting very low cross-coupling of Translational Hand Controler command inputs into other vehicle attitude or translation axes. Many evaluation pilots indicated that the translational pulse sizes were appropriate to the task, though a few desired finer settings. For the Perturbed ISS case, several evaluation pilots noted that the translational pulse size settings were too aggressive and would have preferred a smaller pulse size. Due to the ISS motion and the greater number of inputs required to dock, several pilots noted some attitude dead-banding or mild coupling in this case. Evaluation pilots noted this task as more challenging than the stable ISS case, but still very achievable. Nonetheless, median values for both cases were within Cooper Harper Level 1, each with only a single rating (out of 10) in the Level 2 category. As can be seen in Figure 10, the Perturbed ISS case had a higher workload than the Stable ISS case, but overall, the workload for both cases was very low. 


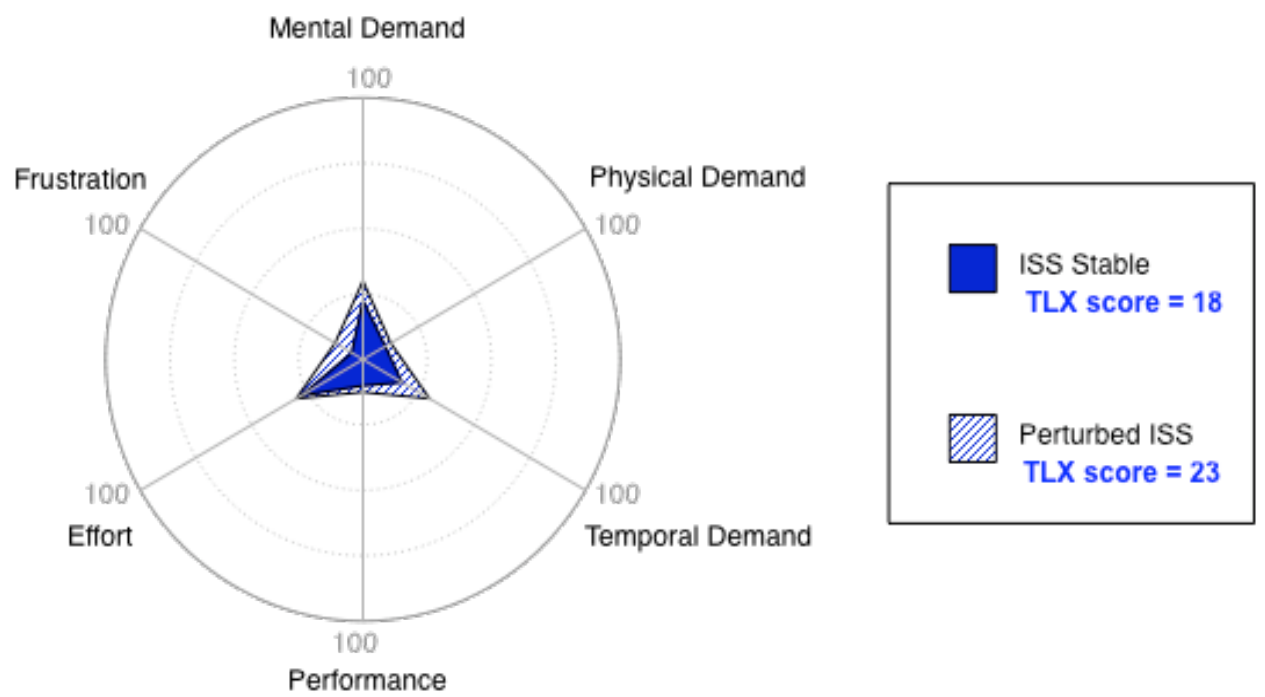

Figure 10. Task Load Index component ratings for docking task

The ProxOps scenario results showed a solid Level 1 rating with every evaluation pilot meeting the desired Cooper-Harper criteria for every run. Evaluation pilots commented on the simplicity of performing this task. No handling qualities issues were uncovered during the evaluation. Two comments indicated that a narrower camera field of view (FOV) would be useful to monitor the motion of the target vehicle and to help determine the direction for nulling Orion's translational rates. Evaluation pilots were only able to use a large FOV, but desired a smaller FOV. This capability will be available on Orion, but was not available during the evaluation. Several evaluation pilots requested a dynamic overlay, an Orion capability not available in this evaluation, showing Orion's relative navigation estimates of the target on the centerline camera view. This would allow the subject to verify relative navigation system's solutions were valid. Two evaluation pilots commented that the displays should include THC translation direction information. Several evaluation pilots (more than the two that commented) actuated the THC in a direction opposite from their desired vector. This polarity issue was most common in the y-axis, but would occasionally manifest in the z-axis. Evaluation pilots who commented on this polarity issue stated that their workload was increased because they had to think through which direction to actuate the THC to receive their desired rate. As can be seen in Figure 11, workload was extremely low for this task.

Several comments were made that are already incorporated into the flight display prototypes, but were not available for this evaluation. Evaluation pilots requested an attitude deadband box on the camera view and that the time to attitude timer on the PFD be moved or repeated on the Traj display. 


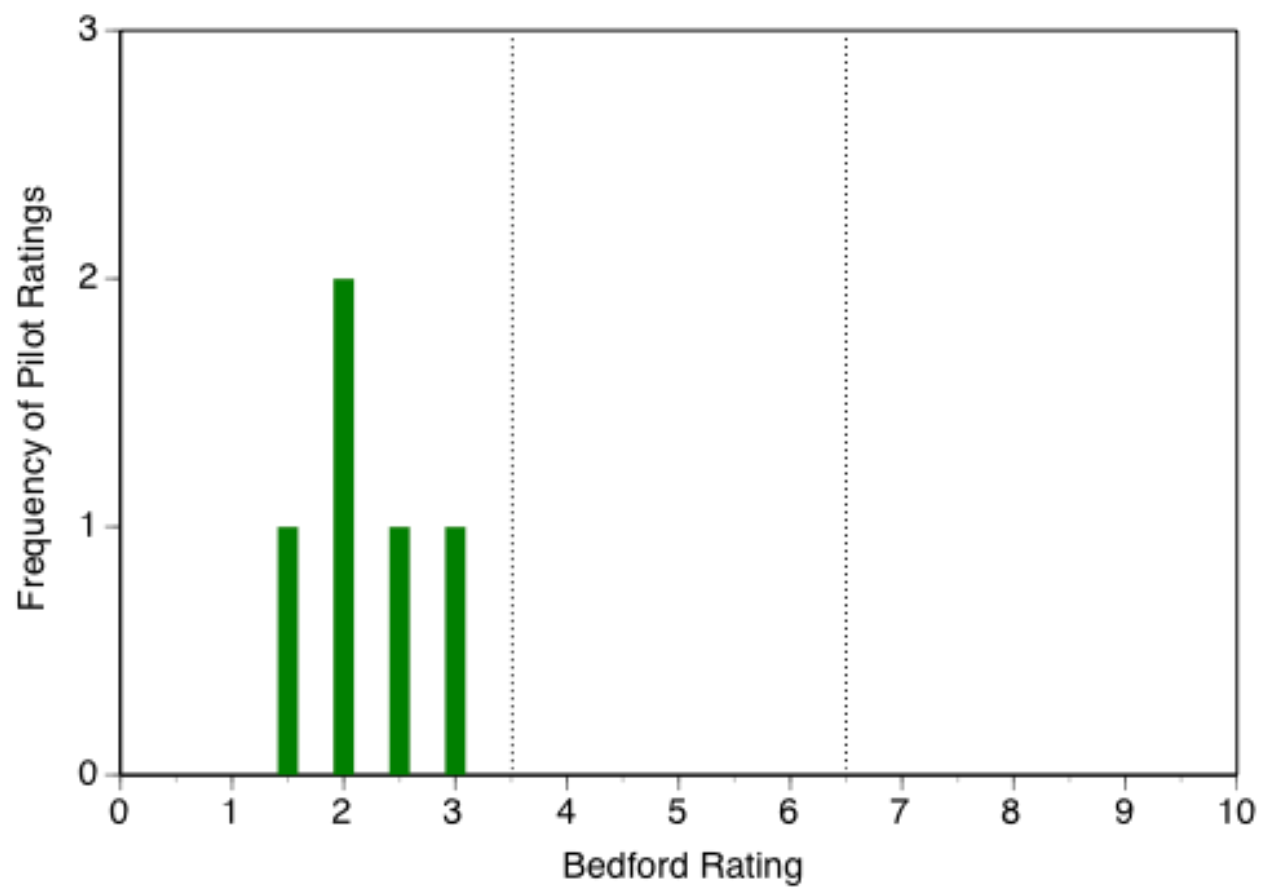

Figure 11. Bedford workload ratings for ProxOps task

\section{Discussion}

The Docking scenario was assessed using both a 'Stable ISS' and a 'Perturbed ISS case. The stable ISS case was included since observed station motion is very minimal, and this case was the most similar to conditions under which the astronauts would actually dock Orion to the ISS. The Perturbed ISS case was based upon the ISS specifications for stability (which date back to a time when the station had fewer modules and much less mass than it does today) which allow a certain degree of motion up to \pm 1 degree of deadband per ISS axis and a $\pm 0.01 \mathrm{deg} / \mathrm{sec}$ rate deadband per ISS axis.

The docking simulation also included several control-related error sources but did not include fuel slosh. Prior to the ProxOps evaluation, the GN\&C team analyzed propellant slosh effects on flight control performance and concluded that propellant slosh had an insignificant affect on flight control. The stable ISS case findings included 9 out of 10 ratings equal or better to a Handling Quality Rating (HQR) of 2, with one rating of 4. The evaluation pilots indicated that there was very low cross-coupling between translation and rotation and that Orion's responses were very predictable, making docking a low workload task.

The Perturbed ISS case was also rated well, with 9 out 10 ratings in the Level 1 category (HQRs of 3 or better) with one rating of 5. In general evaluation pilots rated this scenario one HQR higher (worse) than the stable ISS case, mainly due either to the larger translational pulse sizes (reported as too aggressive) and the ISS attitude deadbanding (not an Orion handling issue). The Perturbed ISS case required more THC inputs and a higher workload than the stable ISS case. Nevertheless, the Perturbed ISS case was reported as being a readily accomplished task.

One evaluation pilot was responsible for both Level 2 ratings for the Docking scenario. The evaluation pilot's comments indicated that better deadband control would lead to a rating of 2 (Level 1) for the Stable ISS case. During the Perturbed ISS case, the evaluation pilot saw the ISS change directions (ISS attitude jets fired to correct the ISS attitude) moments before contact causing the pilot to make a last minute translational correction. While this was not an issue with Orion handling qualities (Orion was able to make the pilot's last second correction satisfactorily), the correction and increased workload impacted the $\mathrm{CH}$ rating. 
In summary, the Docking scenario was generally rated as Level 1 (HQRs of 1-3), for which subject comments indicated that the docking cases were readily achievable. Additional options for pulse sizes and the inclusion of other docking display indicators would make the tasks even more pilotable. These pilot enhancements are available in the actual Orion flight software, but were not available to the evaluation pilots during this evaluation.

The Docking evaluation demonstrated the fine translational control of Orion which is needed to complete docking operations with ISS and the ProxOps evaluation demonstrated the coarse translational control of Orion to arrest the relative motion during acquisition of the docking axis maneuver. No issues with the Service Module RCS translational authority were identified during the ProxOps scenario by evaluation pilots. The ProxOps scenario was rated as a Level 1 task by all evaluation pilots. Most of the subject comments were against the display format prototypes and not the handling of Orion. Probably the most significant comment was to better identify the polarity response of THC inputs, primarily in the y-axis. Several evaluation pilots commented that their workload was increased because they had to contemplate what the vehicle's response polarity would be to an input in a given direction. Many of the other comments are being incorporated into the next iteration of flight displays. Some of these include an attitude deadband box on the centerline camera view, adding a time to desired attitude to the Orbit Traj, adding a 10 degree field of view zoom to the centerline camera, and a dynamic overlay showing Orion's relative navigation's estimate of the target vehicle's location on the centerline camera view.

Overall, the evaluation pilots of this study (all of which were trained NASA astronauts) indicated very minimal cross-coupling between translation and rotation and responses were very predictable, making ProxOps and docking low workload tasks. The tasks evaluated were generally rated as Level 1 indicating that the ProxOps and Docking scenarios were readily achievable with the fine and coarse translational authority provided by Orion. Based upon the reported Cooper-Harper Ratings, subject comments, and additional HQ assessments, Orion's vehicle configuration appears to be on the path to achieving the HQ ratings desired for a human rated spacecraft.

\section{Conclusion}

The goal of the evaluations presented here was to investigate the handling qualities evidenced by NASA's Orion Crew Exploration Vehicle during a variety of rendezvous, proximity, and docking maneuvers. Both the ProxOps scenario as well as the actual docking scenarios demonstrated median Level 1 handling qualities, with all runs resulting in desired performance. Results were additionally associated with low evaluation pilot workload ratings and favorable subjective commentary. Nonetheless, room for improvement was still noted with requested addition of refined translational pulse control modes, and the addition/modification of visual aids represented on the docking displays. These findings have direct relevance to NASA's continued development of the Orion spacecraft as its future deep space mission vehicle, recently renamed the "Multipurpose Crew Vehicle" (MPCV). In its future potential missions to near earth objects or planets such as Mars, Orion may be challenged with additional docking tasks such as rendezvous and docking/undocking with landing vehicles, robotic drones, or long duration spacecraft support systems (e.g. mobile habitat modules). As Orion's mission changes, future handling qualities evaluations will build off of the current study to ensure that Orion maintains its excellent docking handling qualities allowing this most critical of in-space activities to be conducted safely and reliably, ensuring the success of future missions beyond Earth's orbit.

\section{Acknowledgments}

The authors would like to acknowledge all the team members that provided significant contributions on this project to assess handling qualities for Orion RPOD scenarios. Specifically, thanks to Zoran Milenkovic/CSDL, Ed McCants/CSDL, Tom Campbell/ESCG, Chris Foster/ESCG, Justin Thompson/ESCG, Kathryn Sullivan/LM, and Tim Verborgh/AANA for the extraordinary efforts to complete development and verification of the ANTARES simulation and prototype displays under a very tight schedule. Also, thanks to Chad Asuncion/ESCG and Stella Yu/ESCG for efficiently integrating the ANTARES simulation into the ROC and facilitating the assessments with 
the test subjects. Thanks to Jeremy Hart/NASA, Kay Chevray/NASA and Rodolfo Gonzalez/NASA for coordinating and planning the assessments, to Antonius Widjokongko/LM for additional data collection support, and Randy Bresnik/NASA, Jim Dutton/NASA, Pam Melroy/NASA, Lee Archambault/NASA, Phil Root/NASA, Evan Brown/NASA for providing crew office support and coordination.

\section{References}

1. Anon, "Human-Rating Requirements for Space Flight Systems,” NASA Procedural Requirements, NPR 8705.2B, May 2008, pp. 34-35.

2. NASA CxP 70024 Rev D Human-System Integration Requirements

3. Cooper, G.E. and Harper. R.P., "The Use of Pilot Rating in the Evaluation of Aircraft Handling Qualities,” NASA TN D-5153. April 1969.

4. Cheatham, D. C., and Hackler, C. T., "Handling Qualities for Pilot Control of Apollo Lunar-Landing Spacecraft,” Journal of Spacecraft and Rockets, Vol. 3, No. 5, 1966, pp. 632-638. doi:10.2514/3.28506

5. Hackler, C.T., Brickel, J.R., Smith, H.E., and Cheatham, D.C., "Lunar Module Pilot Control Considerations," NASATN D-4131, Feb. 1968.

6. Polites, M. E., “Technology of Automated Rendezvous and Capture in Space,” Journal of Spacecraft and Rockets, Vol. 36, No. 2, 1999, pp. 280-291. doi:10.2514/2.3443

7. Goodman, G. L., "History of Space Shuttle Rendezvous and Proximity Operations," Journal of Spacecraft and Rockets, Vol. 43, No. 5, 2006, pp. 944-959. doi:10.2514/1.19653

8. Burton, J. R., and Hayes, W. E., “Gemini Rendezvous,” Journal of Spacecraft and Rockets, Vol. 3, No. 1, 1966, pp. 145-147. doi:10.2514/3.59526

9. Pennington, J. E., Hatch, H. G., and Driscoll, N. R., “A Full-Size Pilot Controlled Docking Simulation of the Apollo Command and Service Module With the Lunar Module,” NASATN D-3688, Dec. 1966.

10. Riley, D. R., Jaquet, B. M., and Cobb, J. B., "Effect of Target Angular Oscillations on Pilot-Controlled Gemini-Agena Docking,” NASATN D-3403, 1966.

11. Pennington, J. E., Hatch, H. G., Long, E. R., and Cobb, J. B., "Visual Aspects of a Full-Size PilotControlled Simulation of the Gemini- Agena Docking,” NASATN D-2632, 1965.

12. Riley, D. R., Jaquet, B. M., Pennington, J. E., and Brissenden, R. F., "Comparison of Results of Two Simulations Employing Full-Size Visual Cues for Pilot-Controlled Gemini-Agena Docking,” NASATN D3687, Nov. 1966.

13. Hatch, H. G., Pennington, J. E., and Cobb, J. B., "Dynamic Simulation of Lunar Module Docking with Apollo Command Module in Lunar Orbit,” NASATN D-3972, June 1967.

14. Riley, D.R., Jaquet, B.M., Bardusch, R.E., and Deal,P.L., "A Study of Gemini-Agena Docking Using a Fixed-Base Simulator Employing a Closed-Circuit Television System,” NASATN D-3112, 1965.

15. Long, E. R., Pennington, J. E., and Deal, P. L., "Remote Pilot- Controlled Docking With Television," NASATN D-3044, Oct. 1965.

16. Jaquet, B. M., and Riley, D. R., “An Evaluation of Gemini Hand Controllers and Instruments for Docking,” NASATM X-1066, 1965.

17. Zimpfer D. and Spehar P., (1996) “STS-71 Shuttle/Mir GNC Mission Overview,"Advances in the Astronautical Sciences, Vol.93, American Astronautical Society, San Diego, CA, 1996, pp.441- 460; ASS paper 96-129

18. Gibson, R. L., and Precourt, C. J., “The First Space Shuttle to Mir Docking Mission,” Proceedings of the 39thSymposium of the Society of Experimental Test Pilots, Society of Experimental Test Pilots, Lancaster, CA, 1995, pp. 386-419.

19. Acevedo A., Arnold, J., and Othon, W., “ANTARES: Spacecraft Simulation for Multiple User Communities and Facilities,” Paper No. 2007-6888, AIAA Modeling and Simulation Technologies Conference, August 2007. 
20. Gay, R.S., "NASA ANTARES Simulation GNC Architecture,” Paper No. 2007-6679, AIAA Modeling and Simulation Technologies Conference, August 2007.

21. Hart, S.G., and Staveland, L.E., "Development of NASA-TLX: Results of Empirical and Theoretical Research,” in Human Mental Workload, Hancock and Meshkati (Eds.), North Holland Press, Amsterdam, The Netherlands, 1988, pp. 139-183.

22. Roscoe, A.H., “Assessing Pilot Workload in Flight,” Royal Aircraft Establishment (RAE), Bedford, England, 1984.

23. Roscoe, A.H. and Ellis, G.A., "A Subjective Rating Scale for Assessing Pilot Workload in Flight: A Decade of Practical Use,” Royal Aerospace Establishment (RAE) Technical Report TR 90019, 1990. 